\title{
ANÁLISE DAS DIFICULDADES ENCONTRADAS DURANTE A IMPLEMENTAÇÃO DA ENGENHARIA KANSEI
}

João Victor de Azevedo Mataveli (joaomataveli@gmail.com) - Universidade Federal de Itajubá - UNIFEI Carlos Eduardo Sanches da Silva (sanches@unifei.edu.br) - Universidade Federal de Itajubá - UNIFEI Yasmin Silva Martins (yasminsm.sj@gmail.com) - Universidade Federal de Itajubá - UNIFEI

\section{RESUMO}

A Engenharia Kansei é uma técnica de desenvolvimento de produto que permite analisar a percepção e reação dos compradores, satisfazendo suas necessidades implícitas e, tendo como resultados uma melhoria nas vendas, além da fidelização do mercado consumidor.

Existem diferentes métodos para aplicar-se a Engenharia Kansei fato que motivou esta pesquisa, com o objetivo de identificar as dificuldades encontradas durante o processo de implementação da técnica. Foram identificados todos os autores que publicaram artigos ou trabalhos acerca do tema "kansei", por meio de busca nas plataformas Scopus e ISI Web of Science; posteriormente, foram identificadas as etapas do método, perguntando aos autores, por meio de um questionário eletrônico, o grau de dificuldade encontrado para aplicação de cada etapaUm dos fatores que mais dificultaram a pesquisa foi a obtenção de respostas do questionário, devido principalmente, à dificuldade de especificar a população da pesquisa e ao difícil contato com os autores. Conclui-se que, para Nagamachi, criador da Engenharia Kansei, tanto os fatores externos quanto as etapas genéricas possuem baixo grau de dificuldade de implementação; porém, os respondentes não concordam com o autor, indicando que há certo grau de dificuldade em etapas específicas da técnica.

Palavras chave: Engenharia Kansei; Método Kansei; Desenvolvimento de produto 


\section{INTRODUÇÃ̃}

Com o aumento da competitividade e do mercado consumidor, que vem ocorrendo ao longo dos anos, a necessidade de as empresas satisfazerem aos diferentes gostos e estilos dos clientes se torna um grande desafio; além disto, quando se trata do sucesso no desenvolvimento de novos produtos, atender às necessidades dos clientes é considerado um aspecto chave (JIAO e QU, 2019; NAGAMACHI, 1992).

Pensando em resolver esta situação e, assim, conseguir traduzir os anseios dos clientes em parâmetros concretos, surge a Engenharia Kansei.. A técnica desenvolvida pelo professor japonês Matsuo Nagamachi, já foi aplicada a vários setores das indústrias, como no automotivo, máquinas industriais, aplicações elétricas, construções e em grandes empresas como a Mitsubishi, Toyota, Honda, Ford, Delphi e Mazda. Inclusive, a utilização na fabricante de veículos Mazda, resultou no carro Miata, automóvel que alcançou sucesso ao ser lançado nos mercados, como citado por Nagamachi $(1999,2004)$.

Devido ao fato do termo Kansei representar um conceito abstrato, apresentando aspectos da filosofia humana, não é possível medi-lo diretamente, mas pode-se medir suas consequências (NAGASAWA, 1995; NAGAMACHI, 2002). Neste contexto surge a seguinte questão de pesquisa: "quais são as dificuldades encontradas na implementação da Engenharia Kansei?", buscando assim, evidenciar os pontos a serem melhor analisados e que exigem mais atenção.

\section{FUNDAMENTAÇÃO TEÓRICA: KANSEI}

A palavra japonesa Kansei é de difícil tradução direta, devido a seu contexto cultural; no entanto, é comumente interpretada como sensibilidade, sentimento e emoções causadas por algum objeto ou situação. (NAGAMACHI, 1992; ISHIHARA et al., 1993).

A Engenharia Kansei é que uma técnica de desenvolvimento de produtos e serviços que utiliza os conceitos do termo japonês Kansei, analisando a percepção e reação dos compradores, em elementos concretos e atuantes no design dos novos produtos e serviços (NAGAMACHI, 1995).

Apesar do conhecimento sobre a teoria e os possíveis meios de aplicação da técnica, sua aplicação nas empresas pode ser um grande desafio para os engenheiros e toda equipe envolvida no desenvolvimento de novos produtos. Segundo Calantone et al. (1995), a implantação de novas técnicas e tecnologias sempre causarão algum tipo de efeito colateral no âmbito social e 
organizacional das empresas, e isto não é diferente para a implantação da Engenharia Kansei, sendo necessário um trabalho em conjunto entre engenheiros, designers e demais funcionários. Além disto, por ser uma técnica relativamente nova para as empresas, sua implementação requer um suporte contínuo durante o processo (SCHÜTTE, 2002). Esta aplicação pode ocorrer em paralelo ao desenvolvimento dos novos produtos que já ocorre na empresa, oferecendo diretrizes para os produtos futuros, ou ocorrer simultâneamente com os processos já existentes; ou seja, sem o suporte das organizações não seria possível encontrar os melhores pontos do processo onde a Engenharia Kansei pode agir.

Um indício da aprovação da Engenharia Kansei é a quantidade de aplicações que já foram realizadas, autores são comumente chamados a prestar serviços em diversas empresas de setores variados, na indústria automotiva, por exemplo, empresas como Ford, Honda, Nissan e Mazda já utilizaram da técnica. Além disso, o método também conseguiu resultados em setores variados como os de equipamentos domésticos, arquitetura e dispositivos eletrônicos (SCHÜTTE, 2002).

Sobre os aspectos que afetam diretamente o produto ou serviço, Marco-Almagro et al. (2014), exemplificam muito bem a questão implícita existente na interpretação dos clientes quando são expostos a um novo produto. Os autores realizaram um estudo em uma empresa que produzia gabinetes elétricos, os quais satisfaziam todos os padrões de qualidade exigidos por clientes e normas e, apesar disso, não era obtido o sucesso de vendas esperado. Após a realização de pesquisas com os clientes, notou-se que o problema estava na percepção da qualidade que os clientes tinham dos gabinetes, onde pensavam que ele não era robusto o suficiente, mesmo os produtos passando e sendo aprovados por testes de qualidade; os clientes preferiam os produtos das empresas concorrentes por parecerem mais robustos, mesmo que tecnicamente não fossem. Esta pesquisa realizada pelos autores demonstra a utilidade da Engenharia Kansei.

Apesar de existirem métodos variados para a aplicação da Engenharia Kansei, pode ser utilizada uma sequência de etapas padrão, conforme apresentado no

Quadro 1 (SCHÜTTE, 2002; MARCO-ALMAGRO et al., 2014). 
Quadro 1 - Etapas genéricas de aplicação da Engenharia Kansei.

\begin{tabular}{|c|c|}
\hline Etapas & Desdobramentos \\
\hline $\begin{array}{l}\text { Decidir } \\
\text { estrategicamente }\end{array}$ & $\begin{array}{l}\text { Estabelecer os tipos de requisitos que a empresa tem e que possuem potencial de } \\
\text { incorporar maior satisfação para o cliente por meio do desenvolvimento de novos } \\
\text { produtos }\end{array}$ \\
\hline \multirow{2}{*}{$\begin{array}{l}\text { Coletar as palavras } \\
\quad \text { Kansei }\end{array}$} & Coletar palavras Kansei relacionadas ao domínio do produto \\
\hline & $\begin{array}{l}\text { Reduzir as palavras Kansei para um pequeno número de palavras importantes e } \\
\text { relevantes }\end{array}$ \\
\hline \multirow{2}{*}{$\begin{array}{l}\text { Desenvolver uma } \\
\text { escala de medição }\end{array}$} & Desenvolver uma escala capaz de mensurar a resposta do cliente \\
\hline & Selecionar o tipo de escala a usar $(5,7,9$ ou 11 pontos $)$ \\
\hline $\begin{array}{l}\text { Coletar amostras de } \\
\text { produtos }\end{array}$ & Reunir produtos semelhantes ao produto alvo (cerca de 20 ou 25 produtos) \\
\hline $\begin{array}{l}\text { Fazer uma lista de } \\
\text { itens/categorias }\end{array}$ & Separar itens e categorias de características do produto \\
\hline \multirow{2}{*}{$\begin{array}{c}\text { Avaliar o } \\
\text { experimento }\end{array}$} & Selecionar os critérios para avaliar cada amostra \\
\hline & Realizar a avaliação do experimento \\
\hline \multirow{4}{*}{$\begin{array}{l}\text { Realizar as análises } \\
\text { estatísticas }\end{array}$} & Aplicar técnicas e ferramentas estatísticas \\
\hline & Identificar o elemento de design relevante para cada emoção específica \\
\hline & Interpretar os dados \\
\hline & Integrar os dados nas propriedades de design do produto \\
\hline \multirow{4}{*}{$\begin{array}{l}\text { Explicar a } \\
\text { interpretação dos } \\
\text { dados ao designer }\end{array}$} & Colaborar com o designer de produto \\
\hline & Explicar os dados analisados e sua interpretação para o designer \\
\hline & Motivar e estimular o designer a entender a interpretação final dos dados \\
\hline & $\begin{array}{l}\text { Conceber a nova ideia de design do designer incorporando dados emocionais para } \\
\text { além dos dados }\end{array}$ \\
\hline $\begin{array}{l}\text { Conferir a nova } \\
\text { ideia de design }\end{array}$ & $\begin{array}{l}\text { Avaliar se o produto recém-projetado afetará a emoção do cliente e se ele revela o } \\
\text { design emocional }\end{array}$ \\
\hline
\end{tabular}

Fonte: Adaptado de Schütte (2002)

Vale ressaltar que o desenvolvimento de novos produtos apresenta fatores externos à técnica em si que influenciam em seu sucesso, são eles: ter apoio da alta direção da empresa; ter apoio do gerente de projetos; dispor dos recursos e do tempo necessários; ter o compromisso dos membros da equipe; ter conhecimento/capacitação em Engenharia Kansei; ter opiniões conflitantes dentro da equipe; ter conhecimento técnico quanto ao tipo de produto a ser desenvolvido; dispor de uma estrutura de apoio na empresa; ter foco no projeto (MONTOYAWEISS e CALANTONE, 1995; CALANTONE, VICKERY e DROGE, 1995; LAM e CHIN, 2005).

\section{COLETA E ANÁLISE DOS DADOS}

Tendo como termo de busca a palavra "Kansei", selecionando-se o idioma inglês, artigos de periódicos e congressos, em março de 2019 foi realizada uma busca nas plataformas Scopus e 
ISI Web of Science, devido a estas bases possuírem maior número de periódicos e de artigos indexados (MONGEONe PAUL-HUS, 2016). Uma análise preliminar identificou que o criador do método, Nagamachi, é o autor com mais publicações sobre o tema (28 publicações) e que os países que mais publicam são asiáticos.

Foram identificados 154 autores, bem como seus respectivos e-mails, por meio das publicações encontradas nas bases de dados; desta forma, verificou-se a oportunidade de contatá-los, de forma a coletar informações acerca das dificuldades na implementação da Engenharia Kansei, objetivo desta pesquisa. Do total de 154 autores encontrados, 15 (9,74\%) não tiveram seus emails identificados e 27 (17,53\%) os e-mails enviados retornaram com mensagem de erro. Assim, considerou-se 121 autores habilitados a responderem ao questionário, sendo que 5 alegaram não se enquadrar no perfil de respondentes, pois tiveram pouco conhecimento quanto a aplicação do método Kansei, culminando em uma população de 116 autores.

Um questionário, no idioma inglês, foi formatado e disponibilizado para respostas on-line. $\mathrm{O}$ questionário está, de forma resumida, estruturado em:

- Breve explicação da pesquisa: apresentação dos pesquisadores; a quem era destinada a pesquisa; seu propósito; e o tempo estimado para resposta.

- Perfil do respondente: você já participou de algum uso da Engenharia Kansei para desenvolver algum produto/serviço? Quantos? Qual(is) empresa(s)? Qual foi sua função nestes projetos de uso da Engenharia Kansei?

- Dificuldades externas ao método Kansei;

- Dificuldades nas etapas genéricas prescritas no método Kansei;

- Perguntas: você gostaria de fazer algum comentário? (pergunta aberta) e você gostaria de receber um relatório com os resultados desta pesquisa?

Para a classificação das dificuldades externas optou-se por uma escala likert de 7 pontos tendo como os extremos; "1 não dificulta" e "7 dificulta significativamente". E a questão a ser avaliada pelos autores foi: dos fatores externos listados qual sua opinião, fundamentado em sua experiência, da influência deste fator para implementação do método Kansei?

Para a classificação das dificuldades para implementação das etapas do método optou-se por uma escala likert de 7 pontos tendo como os extremos; "1 nenhuma dificuldade" e "7 extremamente difícil”. A questão a ser avaliada pelos autores foi: das etapas genéricas do 
método Kansei qual sua opinião, fundamentado em sua experiência, da dificuldade de implementa-la?

Para minimizar a possibilidade dos e-mails enviados aos respondentes serem classificados como spam, os mesmos foram enviados em grupos de dez e-mails. Semanalmente era verificada a taxa de resposta, sendo os respondentes retirados da listagem de e-mails e novos envios realizados. Após 12 envios semanais, sendo que os dois últimos não tiveram nenhuma resposta, decidiu-se interromper o envio e analisar os 16 respondentes (do total de 116, ou seja, 13,80\%). Os gráficos descritos na Figura 1 apresentam o perfil dos respondentes. Vale destacar que um deles foi o próprio criador do método, Prof. Nagamachi, que descreve mais de 50 aplicações da Engenharia Kansei em mais de 50 empresas, atuando na função de líder da equipe.

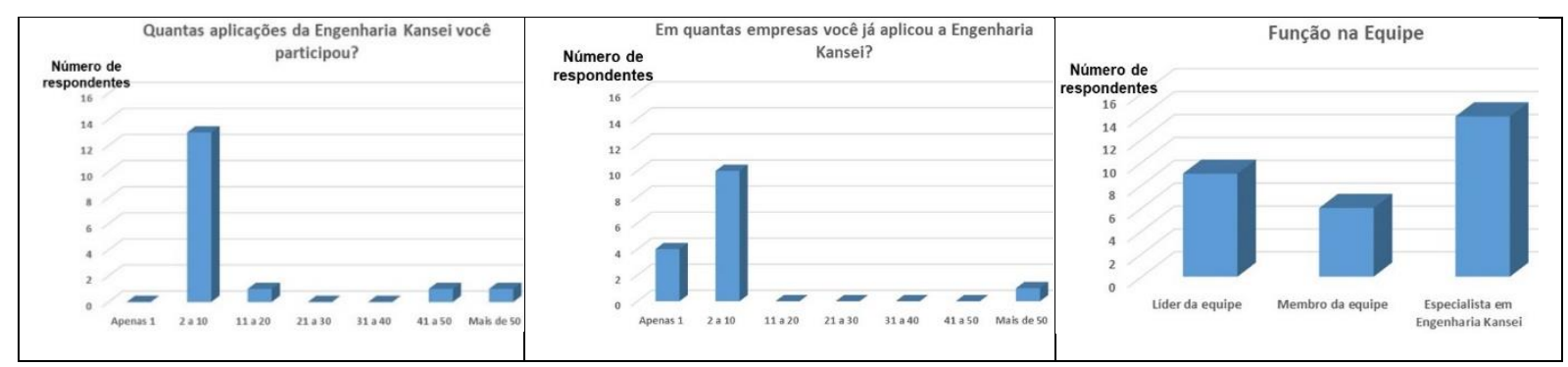

Figura 1 - Perfil dos Respondentes.

A análise dos dados da Figura 1permite validar os respondentes; apesar da grande maioria não ter participado de muitas implementações da Engenharia Kansei, todos possuem domínio do método. As empresas descritas pelos respondentes com implantação da Engenharia Kansei são: Sharp, Komatsu, Panasonic, P\&G, J\&J e Nestlé.

Os dados coletados estão descritos na Tabela 1 e seu bloxplot na Figura 2. Os respondentes foram ordenados pelo número de projetos de Engenharia Kansei realizados. A análise do boxplot da Figura 2 permite identificar que nenhum dos fatores externos apresenta grau de dificuldade elevada, o de maior mediana é "dispor de recursos necessários", seguido dos fatores: "apoio da alta direção"; "dispor de tempo"; e "opiniões conflitantes". O de menor grau de dificuldade foi o "compromisso dos membros da equipe". Estes resultados são semelhantes aos identificados nas pesquisas de Lam e Chin (2005), com valores menores das medianas, exceto para o fator "opiniões conflitantes", que apresentou grau de importância menor. 
Observa-se que todos os fatores apresentaram elevada dispersão, potencialmente devido ao perfil variado dos correspondentes, de iniciantes a experientes na aplicação da técnica.

Tabela 1 - Dados respondentes fatores externos implementação da Engenharia Kansei.

\begin{tabular}{|c|c|c|c|c|c|c|c|c|c|c|c|}
\hline 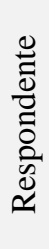 & 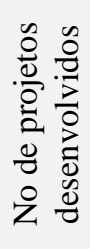 & 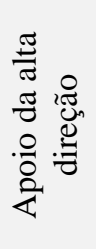 & 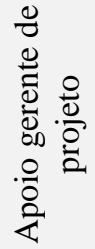 & 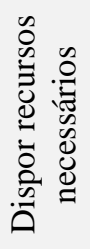 & 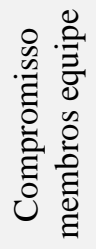 & 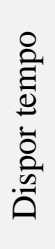 & 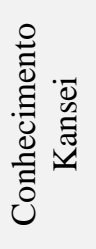 & 导 & 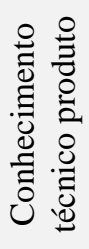 & 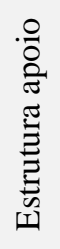 & $\begin{array}{l}\frac{0}{0} \\
\frac{0}{0} \\
0 \\
0 \\
0 \\
0\end{array}$ \\
\hline 1 & 50 & 1 & 1 & 1 & 1 & 4 & 1 & 2 & 1 & 1 & 1 \\
\hline 2 & 30 & 4 & 3 & 3 & 1 & 3 & 3 & 3 & 3 & 3 & 3 \\
\hline 3 & 15 & 5 & 2 & 5 & 1 & 2 & 2 & 4 & 5 & 5 & 3 \\
\hline 4 & 10 & 4 & 3 & 5 & 3 & 4 & 5 & 4 & 3 & 5 & 4 \\
\hline 5 & 10 & 5 & 3 & 5 & 5 & 5 & 5 & 3 & 3 & 4 & 5 \\
\hline 6 & 10 & 4 & 4 & 4 & 2 & 4 & 3 & 4 & 3 & 4 & 4 \\
\hline 7 & 10 & 5 & 5 & 4 & 3 & 3 & 4 & 5 & 5 & 5 & 5 \\
\hline 8 & 5 & 5 & 4 & 5 & 5 & 5 & 4 & 5 & 3 & 6 & 4 \\
\hline 9 & 4 & 5 & 5 & 5 & 2 & 4 & 5 & 3 & 1 & 3 & 2 \\
\hline 10 & 4 & 5 & 6 & 2 & 3 & 3 & 5 & 5 & 4 & 3 & 3 \\
\hline 11 & 3 & 3 & 3 & 5 & 3 & 5 & 3 & 2 & 3 & 2 & 4 \\
\hline 12 & 3 & 4 & 4 & 5 & 5 & 5 & 3 & 5 & 4 & 6 & 5 \\
\hline 13 & 3 & 3 & 3 & 2 & 3 & 3 & 5 & 4 & 4 & 3 & 3 \\
\hline 14 & 3 & 1 & 1 & 1 & 2 & 2 & 3 & 3 & 3 & 3 & 2 \\
\hline 15 & 3 & 2 & 2 & 5 & 2 & 6 & 3 & 3 & 5 & 5 & 6 \\
\hline 16 & 3 & 2 & 3 & 3 & 2 & 4 & 3 & 5 & 2 & 3 & 2 \\
\hline
\end{tabular}

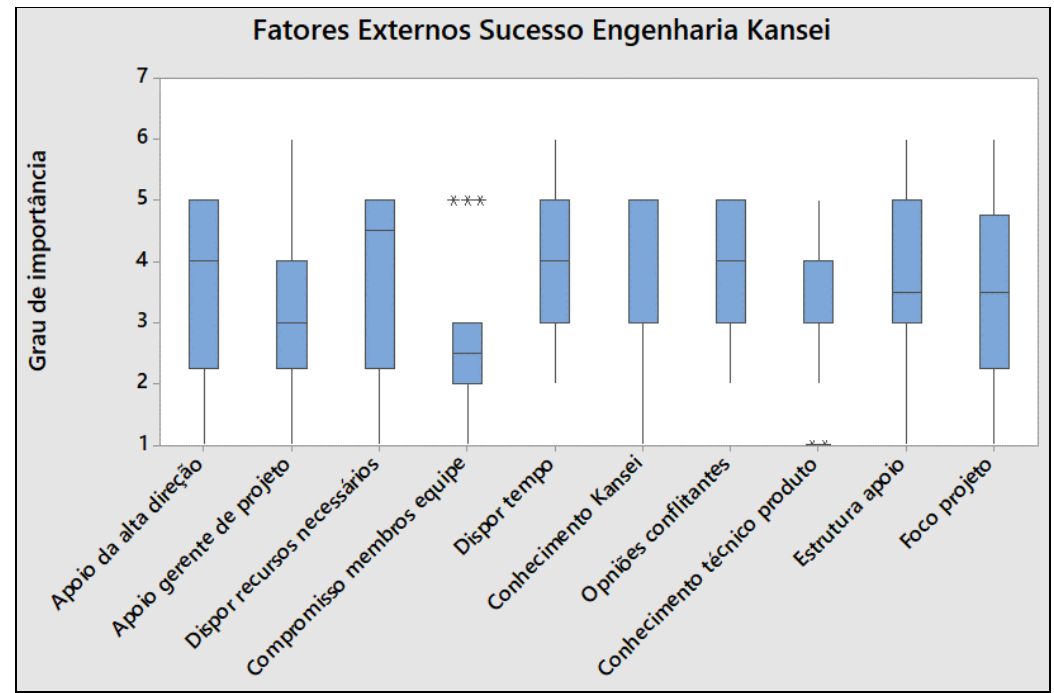

Figura 2 - Fatores externos 
O primeiro respondente é Nagamachi, criador da Engenharia Kansei. Verifica-se na Tabela 1que, para a maioria dos fatores externos, não existe dificuldade de implantação, tendo grau de dificuldade intermediário o item "dispor tempo".

Os dados coletados das etapas genéricas da Engenharia Kansei estão descritos na Tabela 2 e seu bloxplot naFigura 3 .

Tabela 2 - Dados respondentes etapas genéricas da Engenharia Kansei.

\begin{tabular}{|c|c|c|c|c|c|c|c|c|c|c|c|c|c|c|c|c|c|c|c|}
\hline 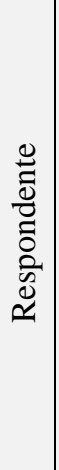 & $\begin{array}{l}0 \\
0 \\
0 \\
0 \\
0 \\
0 \\
0 \\
0 \\
0 \\
0 \\
0 \\
0 \\
0 \\
0 \\
0 \\
0 \\
0 \\
0 \\
0\end{array}$ & 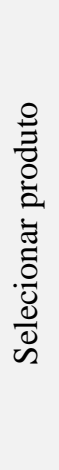 & 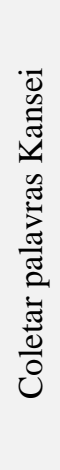 & 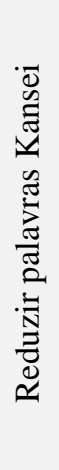 & 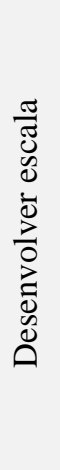 & 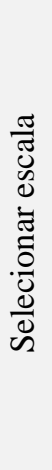 & 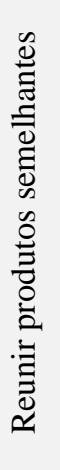 & 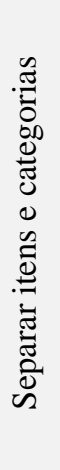 & 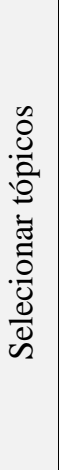 & 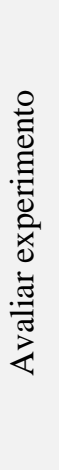 & 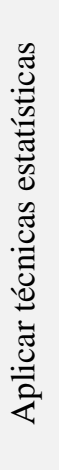 & 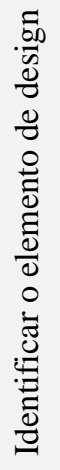 & 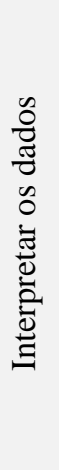 & 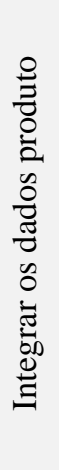 & 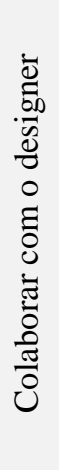 & 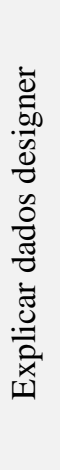 & 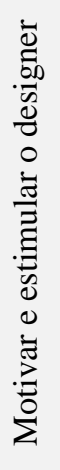 & 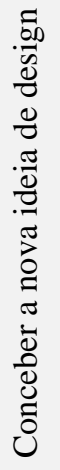 & 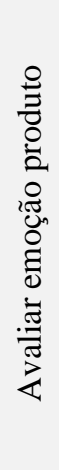 \\
\hline 1 & 50 & 1 & 1 & 1 & 1 & 1 & 1 & 1 & 1 & 1 & 1 & 1 & 1 & 1 & 2 & 1 & 1 & 3 & 1 \\
\hline 2 & 30 & 3 & 1 & 2 & 2 & 5 & 3 & 3 & 4 & 5 & 4 & 4 & 3 & 4 & 3 & 3 & 3 & 2 & 4 \\
\hline 3 & 15 & 5 & 5 & 4 & 5 & 2 & 6 & 6 & 2 & 2 & 3 & 3 & 3 & 3 & 3 & 3 & 4 & 4 & 5 \\
\hline 4 & 10 & 5 & 3 & 3 & 3 & 4 & 5 & 4 & 4 & 5 & 4 & 5 & 5 & 4 & 5 & 4 & 5 & 5 & 4 \\
\hline 5 & 10 & 2 & 2 & 2 & 2 & 1 & 6 & 2 & 2 & 3 & 3 & 3 & 6 & 6 & 4 & 3 & 3 & 3 & 5 \\
\hline 6 & 10 & 4 & 2 & 2 & 2 & 2 & 3 & 5 & 2 & 2 & 2 & 4 & 3 & 5 & 3 & 3 & 4 & 5 & 4 \\
\hline 7 & 10 & 5 & 3 & 3 & 3 & 2 & 3 & 3 & 5 & 5 & 3 & 5 & 3 & 5 & 3 & 4 & 6 & 2 & 4 \\
\hline 8 & 5 & 5 & 4 & 5 & 4 & 3 & 5 & 4 & 4 & 4 & 3 & 4 & 5 & 5 & 5 & 4 & 5 & 5 & 3 \\
\hline 9 & 4 & 2 & 2 & 2 & 1 & 2 & 1 & 2 & 5 & 2 & 6 & 2 & 6 & 3 & 2 & 2 & 6 & 2 & 2 \\
\hline 10 & 4 & 5 & 2 & 3 & 1 & 2 & 3 & 6 & 5 & 5 & 3 & 6 & 6 & 6 & 5 & 5 & 5 & 6 & 5 \\
\hline 11 & 3 & 5 & 5 & 3 & 3 & 3 & 5 & 5 & 5 & 6 & 3 & 3 & 5 & 5 & 5 & 5 & 5 & 5 & 5 \\
\hline 12 & 3 & 5 & 3 & 2 & 2 & 2 & 2 & 6 & 5 & 4 & 3 & 4 & 4 & 5 & 3 & 3 & 4 & 4 & 3 \\
\hline 13 & 3 & 3 & 3 & 3 & 2 & 3 & 5 & 4 & 5 & 3 & 2 & 2 & 3 & 3 & 3 & 4 & 3 & 4 & 4 \\
\hline 14 & 3 & 3 & 2 & 3 & 3 & 3 & 2 & 2 & 2 & 2 & 3 & 3 & 2 & 3 & 3 & 2 & 2 & 2 & 2 \\
\hline 15 & 3 & 6 & 1 & 2 & 1 & 1 & 2 & 2 & 3 & 3 & 1 & 1 & 3 & 5 & 5 & 5 & 5 & 6 & 6 \\
\hline 16 & 3 & 4 & 5 & 6 & 3 & 3 & 6 & 4 & 5 & 6 & 3 & 5 & 2 & 6 & 5 & 6 & 3 & 6 & 7 \\
\hline
\end{tabular}

Nenhuma das etapas da Engenharia Kansei apresenta elevado grau de dificuldade para implementação. A que apresenta dificuldade intermediária é a "seleção do produto", seguida de "separar itens e categorias"; "selecionar tópicos"; "motivar e estimular o designer"; "conceber a nova ideia de design"; e "avaliar a emoção do produto". O fator "desenvolver escala" foi o que apresentou menor grau de dificuldade. Vale destacar que todos os fatores apresentaram elevada dispersão. 


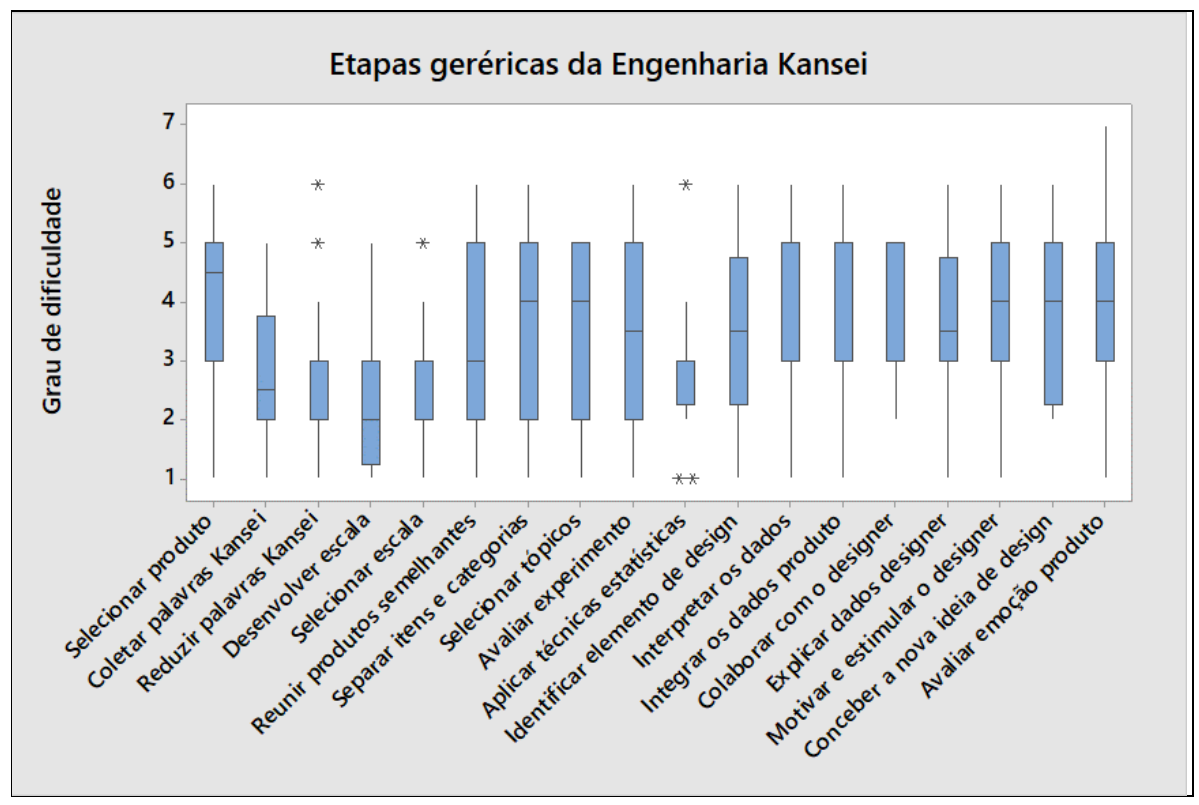

Figura 3 - Etapas da Engenharia Kansei.

A análise de concordância pode ser realizada por meio do coeficiente de Kappa, que é uma medida estatística de concordância utilizada na codificação de informações textuais e na aferição do nível de concordância entre dois ou mais respondentes (BRYMAN e BELL, 2007). O coeficiente de Kappa foi calculado por meio da ferramenta Agreement Analysis do software Minitab 17®, definindo-se como resposta padrão os valores estabelecidos pelo respondente Nagamachi e posteriormente pelo segundo respondente (Tabela 1 e Tabela 2); esta escolha se justifica pelo fato destes respondentes serem os mais experientes. Bryman e Bell (2007) afirmam que o resultado do cálculo deve ser acima de 0,75 para que o coeficiente seja considerado muito bom. Os resultados da análise de concordância estão descritos nasFigura 4Figura 5.

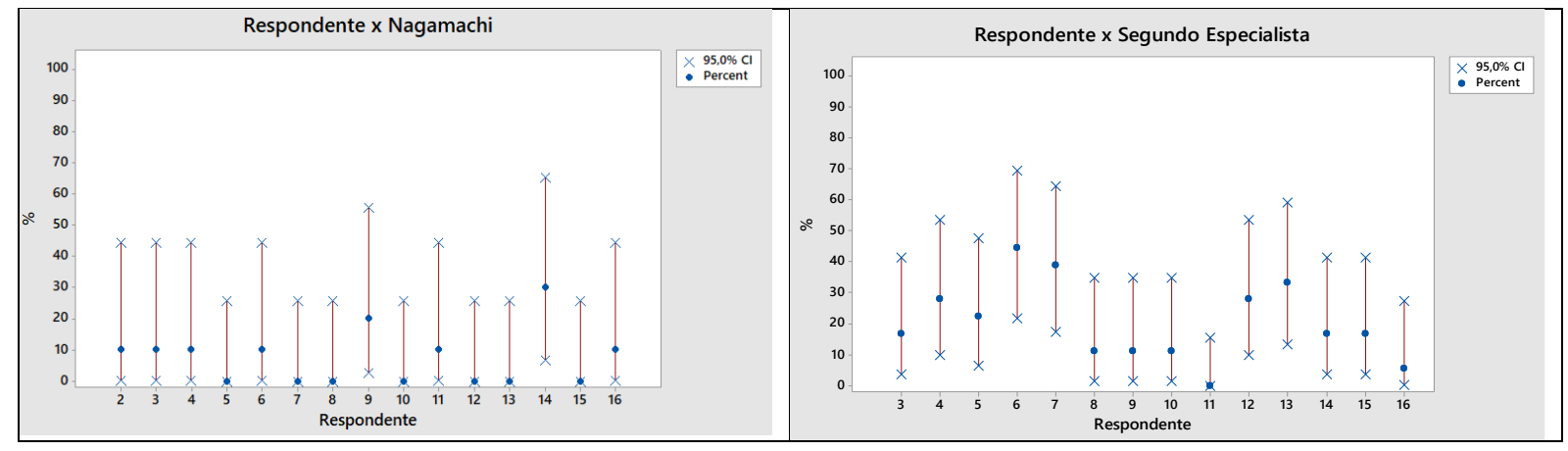


Figura 4 - Analise de concordância entre as respostas de Nagamachi e o segundo respondente em relação aos demais respondentes em relação aos fatores externos para implementação da Engenharia Kansei.

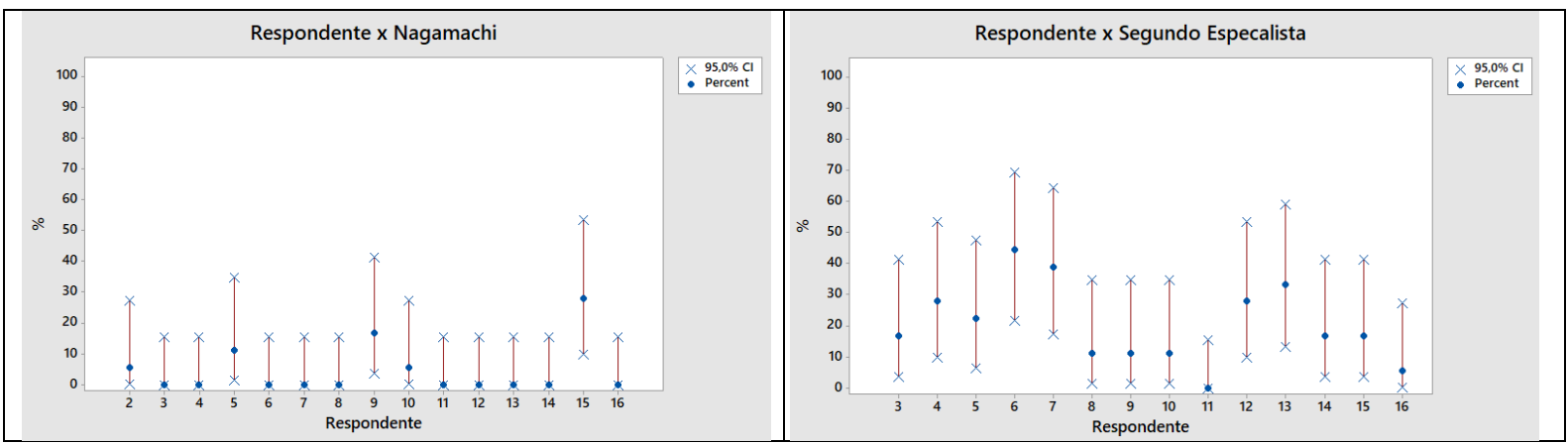

Figura 5 - Analise de concordância entre as respostas de Nagamachi e o segundo respondente em relação aos demais respondentes em relação as etapas para implementação da Engenharia Kansei.

Verifica-se pelos cálculos de concordância a total discordância quanto ao grau de dificuldade, entre os respondentes e o Nagamachi e também entre o segundo respondente em relação aos demais respondentes, para os fatores externos e as etapas genéricas da Engenharia Kansei. Porém, esta discordância é menor entre o segundo respondente e os demais.

Nagamachi considera que quase todos os fatores externos analisados, bem como as etapas genéricas da Engenharia Kansei, em sua maioria, não apresentam dificuldade para implementação, opinião divergente dos demais respondentes. Esta divergência se deve: ao conhecimento técnico e experiência de Nagamachi; às condições pré-estabelecidas para aplicação da Engenharia Kansei junto a alta direção das empresas; e às expectativas da alta administração com os resultados, fato que torna explícitas as necessidades do projeto de aperfeiçoamento do produto, por meio da Engenharia Kansei.

\section{CONCLUSÕES}

Para Nagamachi a dificuldade externa para implementação da Engenharia Kansei é intermediária apenas para a "disponibilidade de tempo", as demais não existem. Já em relação as etapas genéricas para implementação, a única etapa com dificuldade intermediária é “conceber a nova ideia de design". Esta opinião de Nagamachi não é compartilhada com os demais respondentes, que discordam desta análise. Para os demais respondentes os fatores externos que apresentaram a maior mediana foram: "dispor de recursos necessários"; "apoio da alta direção"; "dispor de tempo"; e "opiniões conflitantes". O de menor grau de dificuldade é o 
“compromisso dos membros da equipe". Já em relação as etapas genéricas para implementação da Engenharia Kansei as dificuldades intermediárias são a "seleção do produto"; "separar itens e categorias"; "selecionar tópicos"; "motivar e estimular o designer"; "conceber a nova ideia de design"; e "avaliar a emoção do produto". Já o fator "desenvolver escala" foi o que apresentou menor grau de dificuldade. Não existe nenhum fator externo ou etapa para implementação da Engenharia Kansei, com elevado grau de dificuldade de implementação.

\section{AGRADECIMENTOS}

CAPES, CNPq e FAPEMIG pelo apoio financeiro para realização desta pesquisa.

\section{REFERÊNCIAS}

BRYMAN, A.; BELL, E. Business research methods. $2^{\mathrm{a}}$ ed., New York: Oxford University Press, 2007.

CALANTONE, R.; VICKERY, S.; DROGE, C. Business performance and strategic new product development activities: an empirical investigation. Journal of Product Innovation Management, v. 12, 1995, p. 214-223.

HARADA, A. On the Definition of Kansei. Modeling the Evaluation Structure of Kansei Conference. v. 2, 1988, p. 22.

ISHIHARA, S., ISHIHARA, K., NAGAMACHI, M. Analysis of Individual Differences in Kansei Evaluation Data Based on Cluster Analysis. Kansei Engineering International. 1993, p. 49-58.

JIAO, Y.; QU, Q.-X. A proposal for Kansei knowledge extraction method based on natural language processing technology and online product reviews. Computers in Industry, v. 108, 2019, p. 1-11.

LAM, P.; CHIN, K. Identifying and prioritizing critical success factors for conflict management in collaborative new product development. Industrial Marketing Management, v. 34, 2005, p.761-772.

MARCO-ALMAGRO, L.; TORT-MARTORELL, J. Application of Kansei Engineering to Design an Industrial Enclosure. 14th Annual European Network for Business and Industrial Statistics Conference. Linz: 2014.

MONGEON, P.; PAUL-HUS, A. The journal coverage of Web of Science and Scopus: a comparative analysis. Scientometrics, v. 106(1), 2016, p. 213-228.

MONTOYA-WEISS, M.; CALANTONE, R. Determinants of new product performance: a review and metaanalysis. Journal of Product Innovation Management. v. 111994, p. 397-417.

NAGAMACHI, M. Kansei Engineering And Its Method. Management System, v. 2(2), 1992, p. 97-105.

NAGAMACHI, M. Kansei Enieneering: A new ergonomic consumer-oriented technology for product development. International Journal of Industrial Ergonomics. V. 15, 1995, p. 3-11.

NAGAMACHI, M., Kansei engineering; the implication and applications to product development. Proceedings of the IEEE International Conference on Systems, Man and Cybernetics (VI), 1999, p. 273-278.

NAGASAWA, S. Present State of Kansei Engineering in Japan. 2004 IEEE International Conference. v. 1, 2004, p. 333-338. 
SCHÜTTE, S. Designing Feelings into Products: Integrating Kansei Engineering Methodology in Product Development. Tese (PhD) - Course of Integrating Kansei Engineering Methodology in Product Development, Departament of Mechanical Engineering, Linköpings Universitet, Linköping, 2002, $115 f$.

SCHÜTTE, S., EKLUND, J., AXELSSON, J., \& NAGAMACHI, M. Concepts, Methods and Tools in Kansei Engineering. Theoretical Issues in Ergonomics Science, v. 5(3), 2004, p. 214-231, 